\title{
Floquet Hamiltonians with Pure Point Spectrum
}

\author{
P. Duclos ${ }^{1}$, P. Šťovíček ${ }^{2}$ \\ ${ }^{1}$ Centre de Physique Théorique, CNRS, 13288 Marseille-Luminy and PHYMAT, Université de \\ Toulon et du Var, BP 132, F-83957 La Garde Cedex, France \\ 2 Department of Mathematics and Doppler Institute, Faculty of Nuclear Science, CTU, Trojanova \\ 13, 12000 Prague, Czech Republic
}

Received: 13 December 1994/ Accepted: 26 July 1995

\begin{abstract}
We consider Floquet Hamiltonians of the type $K_{F}:=-i \partial_{t}+H_{0}+\beta V(\omega t)$, where $H_{0}$, a selfadjoint operator acting in a Hilbert space $\mathscr{H}$, has simple discrete spectrum $E_{1}<E_{2}<\cdots$ obeying a gap condition of the type $\inf \left\{n^{-\alpha}\left(E_{n+1}-E_{n}\right)\right.$; $n=1,2, \ldots\}>0$ for a given $\alpha>0, t \mapsto V(t)$ is $2 \pi$-periodic and $r$ times strongly continuously differentiable as a bounded operator on $\mathscr{H}, \omega$ and $\beta$ are real parameters and the periodic boundary condition is imposed in time. We show, roughly, that provided $r$ is large enough, $\beta$ small enough and $\omega$ non-resonant, then the spectrum of $K_{F}$ is pure point. The method we use relies on a successive application of the adiabatic treatment due to Howland and the KAM-type iteration settled by Bellissard and extended by Combescure. Both tools are revisited, adjusted and at some points slightly simplified.
\end{abstract}

\section{Introduction}

Spectral analysis of Floquet Hamiltonians or, equivalently, Floquet operators [7, 17] is known to be a tool to investigate the dynamical stability of a quantum system at least in the spirit of the RAGE theorem (see e.g. [6]). If $K=-i \partial_{t}+H(t)$ is pure point, then for all initial conditions $\psi_{0}$ in $\mathscr{H}$, the solution $\psi_{t}$ of the Schrödinger equation fulfills $\lim _{a \rightarrow \infty} \sup _{t}\left\|\mathscr{E}(|A|>a) \psi_{t}\right\|=0$, with $\mathscr{E}$ being the spectral measure of an arbitrary self-adjoint operator $A$. Though it has been realized recently that such information is rather incomplete; in particular it seems that one cannot predict the time behaviour of $\left\langle\psi_{t}, A \psi_{t}\right\rangle$ from the nature of $\sigma(K)$ (see e.g. [3]).

This paper is concerned with Floquet Hamiltonians $K_{F}:=-i \partial_{t}+H_{0}+\beta V(\omega t)$ depending on two real parameters $\beta$ and $\omega$. The unperturbed (true) Hamiltonian $H_{0}$ in a Hilbert space $\mathscr{H}$ has a simple discrete spectrum $\sigma\left(H_{0}\right)=\left\{E_{1}, E_{2}, \ldots\right\}$ obeying the gap condition given in (2.1) below (with $\alpha>0$ ). The family $V(t)$ is $2 \pi$-periodic and sufficiently many times strongly differentiable.

Except for the methods based on randomizing of some parameter $[8,4]$ and using the Kotani's trick $[13,16]$, two approaches are known to analyze the spectrum of $K_{F}$. The first one is called here the KAM-type iteration method and it was introduced and popularized by Bellissard [1]. This method requires some kind 
of exponential-like decay of matrix entries of the perturbation $V$ if expressed in the eigen-basis of the unperturbed Floquet Hamiltonian $K_{F, 0}:=-i \partial_{t} \otimes 1+1 \otimes H_{0}$. These constraints were afterwards reduced by Combescure [5] to a sufficiently fast power decay. With this hypothesis and assuming in addition that $\beta$ is small enough and $\omega$ non-resonant, one can show that $K_{F}$ is pure point. As observed by Howland [8], another type of results can be obtained using the adiabatic analysis. No restrictions are imposed on $\beta$ and $\omega$ but the information about the spectrum is less precise. If $V(t)$ is smooth enough then the absolutely continuous spectrum of $K_{F}$ is empty.

The present paper is based on the observation that these two methods can be applied successively. First the adiabatic algorithm is used several times to improve the behaviour of the perturbation so that the hypothesis of the KAM iteration method is satisfied. The main theorem is stated in Subsect. 4.1 and claims, roughly, that provided $V(t)$ is sufficiently smooth, $\beta$ sufficiently small and $\omega$ non-resonant, then $K_{F}$ is pure point. As an example we recall in Subsect. 4.2 the well-known quantum Fermi accelerator. Up to now, the adiabatic analysis of this model excluded the absolutely continuous spectrum of the Floquet Hamiltonian $[8,9,15]$. Here we are able to show that the spectrum is even pure point provided the amplitude of oscillations is small and the frequency non-resonant. The mentioned theorem is more or less an immediate consequence of extensive preparatory work contained in Sects. 2 and 3 which was necessary to adjust both the KAM iteration and adiabatic tools to our goal.

Section 2 is devoted to Combescure's modification of the KAM iteration method [5]. The basic difference is that Combescure, wishing to treat the case $\alpha=0$, imposed some additional decay conditions on the entries of the perturbation matrix. We restrict ourselves to $\alpha$ strictly positive and thus we don't employ these constraints. As a consequence we differ in some estimates, orders of decay and constants.

In addition, we modify very slightly the algorithm by not insisting on the full diagonalization in each intermediate step when one adds a part $V^{(n)}$ of the perturbation containing only finitely many parallels to the diagonal. We believe that this makes the structure of the method more transparent. For the same reason we reinterpret one key estimate due to Bellissard [1] as a bound on the norm of an operator $\Gamma$ which inverts the commutation equation $[D, W]=V$, i.e., $\Gamma=\operatorname{ad}_{D}^{-1}$.

Section 3 is devoted to the adiabatic method. Basically, main features of the approach we have used were already contained in Howland's original paper [8]. However there are some differences. To measure the degree of compactness of the perturbation, Howland introduced subspaces of the space of bounded operators by requiring, in principle, a power decay independently in both indices $n$ and $m$ for the matrix $\left(X_{n m}\right)$ of a bounded operator $X$ in a suitable basis. The subspaces introduced in our approach are characterized by a power decay in the absolute value of differences $|n-m|$. They form even subalgebras and seem to fit more naturally with the adiabatic algorithm making the mechanism more transparent and, this is the main reason, they are adjusted to the desired application of the KAM iteration procedure.

The result due to Howland about the absence of the absolutely continuous part of spectrum was generalized by several authors who wished to treat also multiple eigen-values $[14,10]$. These authors applied much more sophisticated machinery. As already mentioned, we restrict ourselves to simple eigen-values but we suggest that this restriction is not intrinsic to the approach we have chosen. We believe that owing to the proper choice of the classes of operators we are able to stay on 
a more elementary level and consequently to treat the problem in a comparatively simple manner.

\section{The KAM-Type Iteration Method}

2.1. Formal Algorithm. We consider a time dependent Hamiltonian $H(t)$ in a separable Hilbert space $\mathscr{H}$ of the type:

$$
H(t):=H_{0}+V(t) .
$$

$H_{0}$ is a selfadjoint operator on $\mathscr{H}$ the spectrum of which is discrete, $\sigma\left(H_{0}\right)=$ $\left\{E_{n} ; n \in \mathbb{N}\right\}$ (remark: $0 \notin \mathbb{N}$ ), and obeys the following fundamental gap condition:

$$
\exists \alpha>0 \text { such that } \inf _{n \in \mathbb{N}} n^{-\alpha}\left(E_{n+1}-E_{n}\right)>0 .
$$

Hence the spectrum $\sigma\left(H_{0}\right)$ is simple. Set also

$$
\Delta E:=\min \left\{E_{n+1}-E_{n} ; n \in \mathbb{N}\right\} .
$$

The function $t \mapsto V(t)$ is bounded measurable and $T$-periodic with values in the symmetric bounded operators in $\mathscr{H}$. The so-called Floquet Hamiltonian $K_{F}:=$ $-i \partial_{t}+H(t)$ acts in $L^{2}(0, T) \otimes \mathscr{H}$. We wish to consider the frequency $\omega=2 \pi / T$ as a parameter lying in a compact interval $\Omega:=[a, b], 0<a<b<\infty$. We found it most convenient to rescale the time so that the period is fixed to be $2 \pi$ and then $\omega$ appears as a parameter standing in front of the time derivative. So from now on,

$$
K_{F}:=-i \omega \partial_{t}+H(t),
$$

acting in $\mathscr{K}:=L^{2}(0,2 \pi) \otimes \mathscr{H}$ with periodic boundary condition in time (and $V(t)$ is assumed to be $2 \pi$-periodic). If the potential $V$ is zero then $K_{F, 0}:=-i \omega \partial_{t} \otimes 1+$ $1 \otimes H_{0}$; its spectrum is simply the closure of $\omega \mathbb{Z}+\sigma\left(H_{0}\right)$. Notice that from the simple form of $K_{F, 0}$ one deduces immediately that it is selfadjoint and since $V$ is bounded and symmetric the same holds true for $K_{F}$.

We shall use the eigen-basis basis of $K_{F, 0}$ to identify the Hilbert space $\mathscr{K}$ with $l^{2}(\mathbb{Z} \times \mathbb{N})$. Thus $K_{F}$ becomes

$$
K \equiv K_{\omega}:=D_{0}+V, \quad \text { acting in } l^{2}(\mathbb{Z} \times \mathbb{N}),
$$

where $D_{0}$ is a diagonal matrix with elements on the diagonal

$$
D_{0}(j) \equiv D_{0}(j ; \omega):=j_{1} \omega+E_{j_{2}},
$$

and $V$ denotes the matrix obtained from the previous function $t \mapsto V(t)$ by computing its matrix elements in the eigen-basis of $K_{F, 0}$.

The method for proving that $K$ has also a pure point spectrum is simply to try to construct a basis of $l^{2}(\mathbb{Z} \times \mathbb{N})$ in which $K$ is diagonal. The algorithm consists in generating a sequence of operators $K_{n}$ which converges to a diagonal operator unitarily equivalent to $K$. To this end we split, following Combescure, the operator $V$ in a sum, with the $n^{\text {th }}$ term $V^{(n)}$ being made of all the elements of the matrix $V$ which are at the distance $n \geqq 0$ from the diagonal:

$$
V=\sum_{n=0}^{\infty} V^{(n)}, \quad V^{(n)}(j, k)=V(j, k) \quad \text { if }|j-k|=n \text { and } 0 \text { otherwise }
$$


where $|j-k|:=\left|j_{1}-k_{1}\right|+\left|j_{2}-k_{2}\right|$. It is easy to see that $V^{(n)}, n \geqq 1$, contains at most $4 n$ non-vanishing parallels to the diagonal. In addition to the sequence $\left\{K_{n}\right\}$, three other auxiliary sequences of matrices are constructed, namely $\left\{G_{n}\right\},\left\{V_{n}\right\}$ and $\left\{W_{n}\right\}$, such that

$$
K_{n}=D_{0}+G_{n}+V_{n}, \quad n=0,1,2, \ldots,
$$

and $G_{n}$ is diagonal for every $n, G_{n}(i, j)=G_{n}(i) \delta_{i j}$, while $V_{n}$ is offdiagonal.

These sequences are determined recursively by the following rules:

(i) $G_{0}=V^{(0)}, V_{0}=0$.

(ii) Provided $G_{n}, V_{n}$ were already determined, then $W_{n}$ solves the equation

$$
\left[D_{0}+G_{n}, W_{n}\right]=V_{n} \quad \text { and } \quad \operatorname{diag} W_{n}=0 .
$$

Hence for $i \neq j$,

$$
W_{n}(i, j)=\frac{V_{n}(i, j)}{D_{0}(i)+G_{n}(i)-D_{0}(j)-G_{n}(j)} .
$$

Clearly, $W_{0}=0$. Uniqueness of the solution of (2.4) makes it possible to define the linear operator $\Gamma_{n}$,

$$
\Gamma_{n} V_{n}:=W_{n}
$$

(iii) Set inductively,

$$
U_{0}:=\mathbf{I}, \quad U_{n}:=\exp \left(W_{n}\right) U_{n-1}=\exp \left(W_{n}\right) \cdots \exp \left(W_{1}\right) .
$$

Then

$$
K_{n+1}:=\mathrm{e}^{W_{n}}\left(D_{0}+G_{n}+V_{n}\right) \mathrm{e}^{-W_{n}}+U_{n} V^{(n+1)} U_{n}^{-1},
$$

and

$$
G_{n+1}:=\operatorname{diag} K_{n+1}-D_{0}, \quad V_{n+1}:=\operatorname{offdiag} K_{n+1} .
$$

Clearly, $G_{1}=V^{(0)}$ and $V_{1}=V^{(1)}$.

We have used above the obvious notation: $\operatorname{diag} A$ for the $\operatorname{diagonal}$ part of $A$ and offdiag $A$ for its off diagonal part.

Finally we make a simple but important observation. The matrix $V(i, j)$ depends on the indices $i_{1}$ and $j_{1}$ only through the difference $i_{1}-j_{1}$, since $V$ acts as a multiplication in the $t$ variable. We claim that the same property is shared by all the matrices $V_{n}(i, j)$ and $W_{n}(i, j)$ and that the diagonal values $G_{n}(i)$ depend only on $i_{2}$ (and not on $i_{1}$ ). Since this property is preserved under multiplication of matrices, to check this claim it suffices to notice that: (i) all the matrices $V^{(n)}(i, j)$ depend only on $i_{1}-j_{1}$, (ii) the diagonal elements $D_{0}(j)$ are linear in $j_{1}$, (iii) the matrices $\left[W, D_{0}\right]$ as well as $e^{W} D_{0} e^{-W}-D_{0}$ depend only on $i_{1}-j_{1}$ provided the same is true for $W$. The claim follows then by induction using the formulas (2.5), (2.7) and (2.8).

2.2. Small Divisor Problem and the $\Gamma$ Operator. The crucial problem when applying the algorithm of the KAM iteration method is the treatment of small divisors in (2.5). Propositions concerned with this difficulty are recalled below. They will be used repeatedly and this is why they are given in a formulation which is independent of the step number. Thus in this subsection we drop the index $n$. In Theorem 2.3 
we shall point out that a key estimate giving a solution to this problem can be interpreted as a bound on the norm of the operator $\Gamma$. But first one has to remove some "bad" or "resonant" values of $\omega \in \Omega=[a, b], 0<a<b<\infty$, which spoil the inequality

$$
\left|\left(D_{0}+G\right)(i)-\left(D_{0}+G\right)(j)\right| \geqq \gamma|i-j|^{-\sigma},
$$

for $\gamma>0$ and $\sigma>0$ chosen in an appropriate way.

Let us formulate it more precisely. Assume that we are given a function $E: \mathbb{N} \rightarrow \mathbb{R}$ fulfilling the $\alpha$-gap condition (2.1) and another function $g: \mathbb{N} \times \mathbb{N} \times$ $\Omega \rightarrow \mathbb{C}$ such that

$$
\begin{aligned}
& \|g\|_{0}:=\sup _{\omega \in \Omega ; n, m \in \mathbb{N}}|g(n, m ; \omega)|<\infty, \\
& \|g\|_{1}:=\sup _{\omega, \omega^{\prime} \in \Omega ; n, m \in \mathbb{N}}\left|\frac{g(n, m ; \omega)-g\left(n, m ; \omega^{\prime}\right)}{\omega-\omega^{\prime}}\right|<\infty
\end{aligned}
$$

and

$$
g(n, m ; \omega)=-g(m, n ; \omega) .
$$

Notice that while $\|\cdot\|_{0}$ is a norm, $\|\cdot\|_{1}$ is only a seminorm. Given $\gamma$ and $\sigma$ positive we set

$$
\Omega_{\mathrm{bad}}:=\bigcup_{\substack{(k, m, n) \in \mathbb{Z} \times \mathbb{N} \times \mathbb{N} \\|k|+|n-m| \neq 0}} I_{k, m, n},
$$

where

$$
I_{k, m, n}:=\left\{\omega \in \Omega ;\left|k \omega+E_{n}-E_{m}+g(n, m ; \omega)\right|<\gamma(|k|+|n-m|)^{-\sigma}\right\} .
$$

The following theorem is due to Bellissard [1] who proved it in the case $\alpha=1$.

Theorem 2.1. Let $E$ and $g$ be defined as above and suppose that

$$
\begin{aligned}
& \|g\|_{0}<\min \{\Delta E, \inf \Omega\}, \\
& \|g\|_{1}<1 .
\end{aligned}
$$

Provided $\sigma$ obeys the inequality $\sigma>\sigma_{\star}(\alpha)$ where

$$
\sigma_{\star}(\alpha):= \begin{cases}\frac{1}{\alpha} & \text { if } 0<\alpha \leqq 1, \\ \frac{2}{1+\alpha} & \text { if } 1<\alpha,\end{cases}
$$

then there exists $C_{1} \equiv C_{1}(E, \Omega, \sigma) \geqq 0$ such that for every $\gamma$,

$$
0<\gamma \leqq \min \{\Delta E, \inf \Omega\}-\|g\|_{0} \Rightarrow\left|\Omega_{\text {bad }}\right| \leqq \frac{C_{1}}{1-\|g\|_{1}} \gamma
$$

For the reader's convenience a sketchy proof is postponed to Appendix 1. It is rather close to Bellissard's original treatment since we can use the fact that $\alpha>0$ and consequently no weights are employed as in [5].

To proceed further we shall need a subalgebra of bounded operators in $l^{2}(\mathbb{Z} \times \mathbb{N})$ distinguished by exponential decay out of the diagonal (matrices are expressed in the standard basis).

Definition 2.2. Banach algebra $\mathscr{B}(\Omega, r), r \geqq 0$, is the subspace of $L^{\infty}\left((\mathbb{Z} \times \mathbb{N})^{2} \times\right.$ $\Omega, \mathbb{C})$ formed by matrices $A$, depending on the parameter $\omega \in \Omega$, for which the 
norm $\|A\|_{\Omega, r}$ is finite $\left(|d|:=\left|d_{1}\right|+\left|d_{2}\right|\right)$,

$$
\begin{aligned}
\|A\|_{\Omega, r}:= & \sum_{d \in \mathbb{Z} \times \mathbb{Z}} \mathrm{e}^{|d| r} \cdot\left(\sup _{i-j=d ; \omega \in \Omega}|A(i, j ; \omega)|\right. \\
& \left.+\sup _{i-j=d ; \omega, \omega^{\prime} \in \Omega}\left|\frac{A(i, j ; \omega)-A\left(i, j ; \omega^{\prime}\right)}{\omega-\omega^{\prime}}\right|\right) .
\end{aligned}
$$

For a diagonal matrix $A,\|A\|_{\Omega, r}$ does not depend on $r$ and we shall sometimes write it also as $\|A\|_{\Omega}$. We shall call $\mathscr{B}^{\text {off }}(\Omega, r)$ the subspace of $\mathscr{B}(\Omega, r)$ of elements having zero diagonals.

Remark. All the properties concerning these algebras are proven in [5].

We are now in a position to recall a theorem which gives a solution to the small divisor problem. In addition to the mapping $E: \mathbb{N} \rightarrow \mathbb{R}$ with the same properties as above we are given a mapping $G: \mathbb{N} \times \Omega \rightarrow \mathbb{C}$ such that, if considered as a diagonal matrix depending on $\omega, G(i, j ; \omega)=G\left(i_{2} ; \omega\right) \delta_{i j}$, $G$ has a finite norm $\|G\|_{\Omega}$. Let finally $\Gamma: \mathscr{B}^{\text {off }}(\Omega, r) \rightarrow \mathscr{B}^{\text {off }}\left(\Omega^{\prime}, r^{\prime}\right)$ be the operator defined by: $W=\Gamma V$ iff $\left[D_{0}+G, W\right]=V$. Here we assume that $0 \leqq r^{\prime}<r, \Omega^{\prime} \subset \Omega$ and $D_{0}$ was given in (2.3).

Theorem 2.3. Let $\Omega, G, E, \Gamma$ and $\sigma>\sigma_{\star}(\alpha)$ be as above and assume in addition that

$$
2\|G\|_{\Omega}<\min \{1, \Delta E, \inf \Omega\} .
$$

Then for every $\gamma$ obeying

$$
0<\gamma \leqq \min \{\Delta E, \inf \Omega\}-2\|G\|_{\Omega},
$$

there exists $\Omega^{\prime} \subset \Omega$ such that

$$
\left|\Omega \backslash \Omega^{\prime}\right| \leqq \frac{C_{1}(\sigma)}{1-2\|G\|} \gamma,
$$

and the norm of $\Gamma: \mathscr{B}^{\mathrm{off}}(\Omega, r) \rightarrow \mathscr{B}^{\mathrm{off}}\left(\Omega^{\prime}, r^{\prime}\right)$ can be estimated by

$$
\|\Gamma\| \leqq C_{2}(\sigma) \gamma^{-2}\left(r-r^{\prime}\right)^{-2 \sigma-1} .
$$

The constant $C_{1}(\sigma) \equiv C_{1}(E, \Omega, \sigma)$ was introduced in Theorem 2.1 and

$$
C_{2}(\sigma) \equiv C_{2}(E, \Omega, \sigma):=\left(\frac{2 \sigma+1}{e}\right)^{2 \sigma+1}(1+\min \{\Delta E, \inf \Omega\}) .
$$

As already mentioned, the bound (2.14) means only a reinterpretation of a known estimate $[1,5]$ and the proof can be found with some modifications in various papers. For example, a nice presentation is given in [2]. But to make this paper self-contained we recall the proof in a sketchy form in Appendix 2.

2.3. Convergence of the Algorithm. Thus it is possible to cope with the small divisor problem provided one accepts losing some values of $\omega$ in each step of the KAM algorithm. In the $n^{\text {th }}$ step, the estimate on the small divisor will be governed by a constant $\gamma_{n}$ and the loss of exponential decay is determined by a constant $\rho_{n}=r_{n}-r_{n+1}$. We choose

$$
\gamma_{n}=\bar{\gamma} n^{-\mu}, \quad \mu>1, \quad \rho_{n}=\bar{\rho} n^{-v}, \quad v>1,
$$


for some fixed positive $\bar{\gamma}$ and $\bar{\rho}$. We introduce also the numbers

$$
r_{n}:=\sum_{j=n}^{\infty} \rho_{j}, \quad \text { hence } r_{n} \leqq \bar{r} n^{-v+1} \text { with } \bar{r}:=\frac{v}{v-1} \bar{\rho}
$$

We set $\Omega_{0}=\Omega_{1}=\Omega$ and then in the $n^{\text {th }}$ step, $n \geqq 1$, we restrict ourselves to $\Omega_{n+1} \subset \Omega_{n}$ so that Theorem 2.3 is applicable. To avoid clumsy notation we shall write simply $\left\|V_{n}\right\| \equiv\left\|V_{n}\right\|_{\Omega_{n}, r_{n}},\left\|W_{n}\right\| \equiv\left\|W_{n}\right\|_{\Omega_{n+1}, r_{n+1}}, \ldots$. To fulfill the hypothesis of Theorem 2.3 we shall assume now and verify afterwards that the norms $\left\|G_{n}\right\|$ are bounded uniformly and

$$
\sup _{n \geqq 1}\left\|G_{n}\right\| \leqq \frac{1}{4} \min \{\Delta E, \inf \Omega, 1\} .
$$

Furthermore we shall require the constant $\bar{\gamma}$ to satisfy

$$
\bar{\gamma} \leqq \frac{1}{2} \min \{\Delta E, \inf \Omega, 1\},
$$

and hence $\bar{\gamma} \leqq \min \{\Delta E$, inf $\Omega\}-2 \sup _{n \geqq 1}\left\|G_{n}\right\|$.

With these restrictions one can actually apply Theorem 2.3 and so $\left|\Omega_{n} \backslash \Omega_{n+1}\right| \leqq$ $C_{1}\left(1-2\left\|G_{n}\right\|\right)^{-1} \gamma_{n}$ and for the operator $\Gamma_{n}: \mathscr{B}^{\text {off }}\left(\Omega_{n}, r_{n}\right) \rightarrow \mathscr{B}^{\text {off }}\left(\Omega_{n+1}, r_{n+1}\right)$ we have $\left\|\Gamma_{n}\right\| \leqq C_{2} \gamma_{n}^{-2} \rho_{n}^{-2 \sigma-1}$. Then for

$$
\Omega^{\prime}:=\bigcap_{n \geqq 0} \Omega_{n},
$$

it is clearly true

$$
\left|\Omega \backslash \Omega^{\prime}\right| \leqq C_{1}(\sigma)\left(1-2 \sup _{n \geqq 1}\left\|G_{n}\right\|\right)^{-1} \gamma, \quad \text { where } \gamma:=\frac{\mu}{\mu-1} \bar{\gamma} \geqq \sum_{1}^{\infty} \gamma_{n} .
$$

Letting

$$
C_{\Gamma}:=\frac{C_{2}(\sigma)}{\bar{\gamma}^{2} \bar{\rho}^{2 \sigma+1}}
$$

the bound on $\Gamma_{n}$ can be rewritten as

$$
\left\|\Gamma_{n}\right\| \leqq F_{n}:=C_{\Gamma} n^{2 \mu+(2 \sigma+1) v} .
$$

Some other estimates follow obviously,

$$
\left\|W_{n}\right\| \leqq\left\|\Gamma_{n}\right\|\left\|V_{n}\right\|, \quad\left\|U_{n}^{ \pm 1}\right\| \leqq \exp \left(\sum_{m=1}^{n}\left\|W_{m}\right\|\right) .
$$

Though the operator $\Gamma$ is defined on the space $\mathscr{B}^{\text {off }}(\Omega, r)$ distinguished by exponential decay the matrix $V$ is required, owing to Combescure's trick, to exhibit only sufficiently fast power decay. We shall characterize it by the quantity

$$
C_{V}:=\sup _{d \in \mathbb{Z}^{2}}\left(1+|d|^{\tau}\right) \sup _{i-j=d}|V(i, j)|<\infty .
$$

The constraints on the power $\tau$ will be specified later. Notice that the norm $\left\|V^{(n)}\right\|_{\Omega, r}$ is finite for any $r \geqq 0$. In fact, since $V^{(n)}$ doesn't depend on $\omega$,

$$
\left\|V^{(n)}\right\|_{\Omega, r} \leqq 4 n \mathrm{e}^{n r} \sup _{|i-j|=n}|V(i, j)|
$$


Setting here $r=r_{n} \leqq \bar{r} n^{-v+1}$ and taking into account (2.18) we get $(n \geqq 1)$,

$$
v \geqq 2 \Rightarrow\left\|V^{(n)}\right\| \leqq 4 C_{V} \mathrm{e}^{\bar{r}} n^{1-\tau} .
$$

Our goal is to show that under appropriate conditions, $\left\|V_{n}\right\| \equiv\left\|V_{n}\right\|_{\Omega_{n}, r_{n}}$ tends to zero as $n$ tends to infinity. It is elementary to derive the following lemma from the relations (2.7) and (2.8) defining the algorithm, provided one uses the fact that $\|\cdot\|_{\Omega, r}$ is a norm of an algebra and the equality $\left[D_{0}+G_{n}, W_{n}\right]=V_{n}$.

Lemma 2.4. For any $n \geqq 1$ one has

$$
\begin{aligned}
&\left\|V_{n+1}\right\| \leqq 2 e^{2\left\|W_{n}\right\|}\left\|W_{n}\right\|\left\|V_{n}\right\|+\left\|U_{n}\right\|\left\|U_{n}^{-1}\right\|\left\|V^{(n+1)}\right\|, \\
&\left\|U_{n}^{ \pm 1}\right\| \leqq e^{\left\|W_{n}\right\|}\left\|U_{n-1}^{ \pm 1}\right\|, \\
&\left\|G_{n+1}-G_{n}\right\|_{\Omega_{n+1}} \leqq 2 e^{2\left\|W_{n}\right\|}\left\|W_{n}\right\|\left\|V_{n}\right\|+\left\|U_{n}\right\|\left\|U_{n}^{-1}\right\|\left\|V^{(n+1)}\right\| .
\end{aligned}
$$

Let us now introduce an auxiliary sequence $\left\{x_{n}\right\}$,

$$
x_{0}:=0 \quad \text { and } \quad x_{n}:=F_{n}\left\|V_{n}\right\|, \quad \text { for } n \geqq 1,
$$

and constants

$$
C_{3}:=\sup _{n \geqq 1} \frac{F_{n+1}}{F_{n}} \leqq 2^{2 \mu+(2 \sigma+1) v}, \quad C_{4}:=4 \mathrm{e}^{\bar{r}} C_{V} C_{\Gamma} .
$$

Multiplying both sides of the inequality (2.19) by $F_{n+1}$ we get for $n \geqq 0$,

$$
x_{n+1} \leqq 2 C_{3} x_{n}^{2} \mathrm{e}^{2 x_{n}}+\frac{C_{4}}{(n+1)^{\beta}} \exp \left(2 \sum_{m=1}^{n} x_{m}\right),
$$

where

$$
\beta:=\tau-1-2 \mu-(2 \sigma+1) v .
$$

It is easy to solve this finite difference inequality.

Lemma 2.5. Assume that $\beta>1$ and a sequence $\left\{x_{n}\right\}_{n \geqq 0}$ obeys the inequality (2.24) and $x_{0}=0$. Then there exists a constant $C_{4}^{\star} \equiv C_{4}^{\star}\left(\beta, C_{3}\right)$ such that $C_{4} \leqq$ $C_{4}^{\star}$ implies

$$
x_{n} \leqq 3 C_{4} n^{-\beta}, \quad \text { for all } n \geqq 1 .
$$

Proof. Clearly, (2.24) implies $x_{1} \leqq C_{4} \leqq 3 C_{4}$. The proof is then easily carried out by induction. $C_{4}$ should be small enough so that the following induction step goes through,

$$
\begin{aligned}
x_{n} \leqq 3 C_{4} n^{-\beta} & \Rightarrow\left\{\begin{array}{ll}
2 C_{3} x_{n}^{2} e^{2 x_{n}} & \leqq C_{4}(n+1)^{-\beta} \\
C_{4}(n+1)^{-\beta} \exp \left(2 \sum_{j=1}^{n} x_{j}\right) & \leqq 2 C_{4}(n+1)^{-\beta}
\end{array}\right\} \\
& \Rightarrow x_{n+1} \leqq 3 C_{4}(n+1)^{-\beta} .
\end{aligned}
$$

The constraints on $\tau$ follow from the requirement $\beta>1$. Since $\mu>1, v \geqq 2$ and $\sigma>\sigma_{\star}(\alpha)$, we get

$$
\tau>2+2 \mu+(2 \sigma+1) v>4+2\left(2 \sigma_{\star}+1\right)=4 \sigma_{\star}+6 .
$$


This means

$$
\tau>\tau_{\star}(\alpha):=\left\{\begin{array}{ll}
6+\frac{4}{\alpha} & \text { if } \alpha \leqq 1 \\
6+\frac{8}{1+\alpha} & \text { if } \alpha>1
\end{array} .\right.
$$

Conversely, if $\tau>\tau_{\star}(\alpha)$ then one can choose $\mu>1, v \geqq 2$ and $\sigma>\sigma_{\star}(\alpha)$ so that $\beta>1$. In that case $C_{3}$ and $C_{4}^{*}$ are fixed by (2.23) and Lemma 2.5 , respectively. According to (2.23), the inequality $C_{4} \leqq C_{4}^{*}$ will be satisfied provided $C_{V}$ is small enough,

$$
C_{V} \leqq \frac{1}{4} \mathrm{e}^{-\bar{r}} C_{4}^{\star} C_{2}(\sigma)^{-1} \bar{\rho}^{2 \sigma+1} \cdot \bar{\gamma}^{2}
$$

We must still verify the condition (2.15) on $\left\|G_{n}\right\|$. From (2.21) and the proof of Lemma 2.5 one obtains immediately that

$$
\begin{aligned}
\left\|G_{n+1}-G_{n}\right\|_{\Omega_{n+1}} & \leqq F_{n+1}^{-1}\left(2 C_{3} x_{n}^{2} \mathrm{e}^{2 x_{n}}+C_{4}(n+1)^{-\beta} \exp \left(2 \sum_{1 \leqq m \leqq n} x_{m}\right)\right) \\
& \leqq 3 C_{4} F_{n+1}^{-1}(n+1)^{-\beta}=12 \mathrm{e}^{\bar{r}} C_{V}(n+1)^{1-\tau} .
\end{aligned}
$$

Consequently, recalling that $G_{1}=V^{(0)}$,

$$
\begin{aligned}
\left\|G_{n}\right\| & \leqq\left\|G_{1}\right\|+\sum_{n=2}^{\infty} 12 \mathrm{e}^{\bar{r}} C_{V} n^{1-\tau} \leqq\left\|V^{(0)}\right\|+\frac{12}{\tau-2} \mathrm{e}^{\bar{r}} C_{V} \\
& \leqq C_{V}\left(1+\frac{12}{\tau-2} \mathrm{e}^{\bar{r}}\right) \leqq C_{V}\left(1+3 \mathrm{e}^{\bar{r}}\right),
\end{aligned}
$$

where in the last line we used the fact that $\tau$ is always greater than 6 . So again, one can always satisfy the bound (2.15) by taking $C_{V}$ small enough.

We have arrived at the conclusion that $\left\|V_{n}\right\|_{\Omega^{\prime}, 0}$ tends to 0 as $n \rightarrow \infty$ (cf. (2.17), (2.22) and (2.26)). By the Cauchy criterion, $G_{n}$ tends to some matrix $G_{\infty}$ in the norm $\|\cdot\|_{\Omega^{\prime}, 0}$ and necessarily $G_{\infty}$ is diagonal. Similarly, since

$$
\sum_{n=1}^{\infty}\left\|W_{n}\right\|_{\Omega^{\prime}, 0} \leqq \sum_{n=1}^{\infty} F_{n}\left\|V_{n}\right\|<\infty
$$

$U_{n}^{ \pm 1}$ tends to $U_{\infty}^{ \pm 1}$ in the same norm.

2.4. Stability of the Pure Point Spectrum. One can show by induction that the relations $(2.7)$ and $(2.8)$ mean $(n \geqq 1)$

$$
D_{0}+G_{n}+V_{n}=U_{n-1}\left(D_{0}+\sum_{m=0}^{n} V^{(m)}\right) U_{n-1}^{-1} .
$$

It is possible to perform the limit $n \rightarrow \infty$ since the convergence in the norm $\|\cdot\|_{\Omega^{\prime}, 0}$ implies the convergence in the standard operator norm in $\mathscr{B}\left(l^{2}\right)$ for any fixed $\omega \in \Omega^{\prime}$ [5]. In addition to the final conclusion of the preceding subsection we note that obviously

$$
\sum_{m=0}^{n} V^{(n)} \rightarrow V, \quad \text { as } n \rightarrow \infty,
$$


in the norm $\|\cdot\|_{\Omega^{\prime}, 0}$ and hence in the standard operator norm as well. However, respecting the fact that $D_{0}$ is unbounded though self-adjoint with its natural domain, let us make a short comment about the convergence procedure since this point is usually carelessly skipped.

First note that $W_{n}$ preserves the domain $D_{0}$ and $\left[D_{0}, W_{n}\right]$ is bounded. To this end it is enough to observe that $D_{0}$, being diagonal, can be applied formally to any sequence (without any restrictions on summability) and it holds formally

$$
D_{0} W_{n}=W_{n}\left(D_{0}+G_{n}\right)-G_{n} W_{n}+V_{n} .
$$

Consequently we find that $\exp \left( \pm W_{n}\right)$ preserves the domain of $D_{0}$ and

$$
\exp \left( \pm W_{n}\right) D_{0} \exp \left(\mp W_{n}\right)-D_{0}
$$

is bounded. This follows from the formal equality

$$
D_{0} \exp \left(W_{n}\right)=\exp \left(W_{n}\right) D_{0}+\sum_{k=1}^{\infty} \frac{1}{k !} \sum_{j=0}^{k-1} W_{n}^{j}\left[D_{0}, W_{n}\right] W_{n}^{k-1-j}
$$

Hence $U_{n}^{-1}$ preserves the domain of $D_{0}$ as well and the equality (2.29) can be rewritten

$$
D_{0} U_{n-1}^{-1}=U_{n-1}^{-1}\left(D_{0}+G_{n}+V_{n}\right)-\left(\sum_{m=0}^{n} V^{(m)}\right) U_{n-1}^{-1} .
$$

Now to perform the limit procedure it is enough to notice that $D_{0}$ is closed. We conclude that $U_{\infty}^{-1}$ preserves the domain of $D_{0}$, too, and

$$
U_{\infty}\left(D_{0}+V\right) U_{\infty}^{-1}=D_{0}+G_{\infty}
$$

We note also, as it is quite obvious from their construction (cf. (2.4), (2.8)) that all the matrices $W_{n}(j, k)$ are anti-Hermitian and the diagonal elements $G_{n}\left(i_{2}\right)$ are real provided the matrix $V(j, k)$ is Hermitian. Consequently, in that case $U_{\infty}$ is unitary.

Thus after an obvious rescaling we get

Theorem 2.6. Let $\Omega=[a, b] \subset] 0, \infty\left[\right.$ and $\Omega \ni \omega \mapsto K_{\omega}:=D_{0}+V$ be a family of selfadjoint operators acting in $l^{2}(\mathbb{Z} \times \mathbb{N})$. $D_{0}$ is supposed to be diagonal in the standard basis of $l^{2}(\mathbb{Z} \times \mathbb{N})$ with entries on the diagonal given by

$$
\forall i \in \mathbb{Z} \times \mathbb{N}, \quad D_{0}(i):=\omega i_{1}+E_{i_{2}},
$$

where $E: \mathbb{N} \rightarrow \mathbb{R}$ fulfills the gap condition

$$
\inf \left\{n^{-\alpha}\left(E_{n+1}-E_{n}\right) ; n \in \mathbb{N}\right\}>0
$$

for some strictly positive $\alpha$. We assume also that $V$ obeys the following power law decay condition

$$
\sup _{d \in \mathbb{Z}^{2}}\left(1+|d|^{\tau}\right) \sup _{i-j=d}|V(i, j)|=: C_{V}<\infty
$$

for some $\tau$ strictly greater than $\tau_{\star}(\alpha)$ given in (2.27). 
Then there exist two constants $\gamma^{\star}>0, C^{\star}>0$ such that the inequalities $0<\gamma<\gamma^{\star}$ and $0 \leqq C_{V} \leqq C^{\star} \gamma^{2}$ imply that one can find a subset $\Omega^{\prime} \subset \Omega$ fulfilling $\left|\Omega \backslash \Omega^{\prime}\right|<\gamma$ and for every $\omega \in \Omega^{\prime}, K_{\omega}$ is pure point.

\section{Adiabatic Method}

3.1. Classes of Operators. The assumptions on the unperturbed Hamiltonian $H_{0}$ and its spectrum $\sigma\left(H_{0}\right)=\left\{E_{1}, E_{2}, \ldots\right\}$, including the $\alpha$-gap condition (2.1) with some $\alpha>0$, are the same as in Sect.2. Using the eigen-basis of $H_{0}$ we identify the Hilbert space $\mathscr{H}$ with $l^{2}=l^{2}(\mathbb{N})$. This means that the eigen-vectors of $H_{0}$ coincide with the vectors of the standard basis $\left\{e_{n}\right\}$ in $l^{2}$. The aim is to modify Howland's adiabatic treatment in order to show that provided $V(t)$ is smooth enough then the Floquet operator $K=-i \partial_{t}+H_{0}+V(t)$ (with periodic boundary conditions in $t$ ) is unitarily equivalent to another one, $\tilde{K}=-i \partial_{t}+H_{0}+\tilde{V}(t)$, and the new perturbation $\tilde{V}(t)$ enables application of the KAM algorithm. In this situation the value of the period is inessential.

We start from the définition of the classes of bounded operators as announced in Introduction.

Definition 3.1. For $k \in \mathbb{Z}_{+}\left(0 \in \mathbb{Z}_{+}\right)$we say that a bounded operator $X$ belongs to the class $\mathscr{A}_{k} \subset \mathscr{B}\left(l^{2}\right)$ if and only if $\left(\mathrm{ad}_{H_{0}}\right)^{k} \cdot X \in \mathscr{B}\left(l^{2}\right)$. Thus by definition $\mathscr{A}_{0}=\mathscr{B}\left(l^{2}\right)$ and we set also $\mathscr{A}_{\infty}=\bigcap_{k \geqq 0} \mathscr{A}_{k}$.

Notice that a matrix $\left(X_{n m}\right)$, if expressed in the standard basis, corresponds to an operator $X \in \mathscr{A}_{k}$ if and only if the diagonal sequence $\left\{X_{n n}\right\}$ belongs to $l^{\infty}$ and the matrix with entries $\left(E_{n}-E_{m}\right)^{k} X_{n m}$ corresponds to a bounded operator. It is so because the last two conditions imply that the matrix $\left(X_{n m}\right)$ itself corresponds to a bounded operator. This assertion can be easily verified using the fact that the $\alpha$-gap condition (2.1) implies

$$
\inf _{n \neq m}\left|n^{1+\alpha}-m^{1+\alpha}\right|^{-1}\left|E_{n}-E_{m}\right|>0 .
$$

Thus it is enough to show the boundedness of the operator $Y$ corresponding to the matrix $\left(Y_{n m}\right), Y_{n n}=0$ and $Y_{n m}=\left|n^{1+\alpha}-m^{1+\alpha}\right|^{-k}$ for $n \neq m(k \geqq 1)$. But since $1 \geqq z^{\beta}+(1-z)^{\beta}$ for any $z \in[0,1]$ and $\beta \geqq 1$, we have $\left|x^{\beta}-y^{\beta}\right| \geqq|x-y|^{\beta}$ for any $x>0, y>0$. Then the Schur-Holmgren criterion gives the result,

$$
\sup _{n} \sum_{m} Y_{n m} \leqq \sup _{n} \sum_{m \neq n}|n-m|^{-(1+\alpha) k} \leqq 2 \sum_{j=1}^{\infty} j^{-(1+\alpha) k} .
$$

Let us summarize basic properties of the classes $\mathscr{A}_{k}$.

Lemma 3.2. The classes $\mathscr{A}_{k}$ are nested,

$$
\mathscr{A}_{k+1} \subset \mathscr{A}_{k}, \text { for } k=0,1, \ldots \text {. }
$$

For every $k, \mathscr{A}_{k}$ is a *-subalgebra in $\mathscr{B}\left(l^{2}\right)$.

Proof. Concerning the property (3.3), for $k=0$ it is true by definition and for $k \geqq 1, X \in \mathscr{A}_{k+1}$ implies that the operator $\left(\mathrm{ad}_{H_{0}}\right)^{k} \cdot X$ with vanishing diagonal belongs to $\mathscr{A}_{1} \subset \mathscr{B}\left(l^{2}\right)$. 
$\mathscr{A}_{k}$ is closed with respect to multiplication because of (3.3) and the relation

$$
\left(\operatorname{ad}_{H_{0}}\right)^{k} \cdot X Y=\sum_{j=0}^{k}\left(\begin{array}{l}
k \\
j
\end{array}\right)\left(\left(\operatorname{ad}_{H_{0}}\right)^{j} \cdot X\right)\left(\left(\operatorname{ad}_{H_{0}}\right)^{k-j} \cdot Y\right) .
$$

$\mathscr{A}_{k}$ is closed with respect to Hermitian conjugation because of

$$
\left(\operatorname{ad}_{H_{0}}\right)^{k} \cdot X^{*}=(-1)^{k}\left(\left(\operatorname{ad}_{H_{0}}\right)^{k} \cdot X\right)^{*} \text {. }
$$

The following definition takes into account the time dependence.

Definition 3.3. We shall say that a family $X(t)$ of bounded operators depending periodically on $t$ (with a given period) belongs to $C^{r}\left(\mathscr{A}_{k}\right)$ if and only if $X(t)$ is strongly $C^{r}$ and $\left(\operatorname{ad}_{H_{0}}\right)^{k} \cdot(d / d t)^{s} X(t)$ is bounded and strongly continuous for $s=0,1, \ldots, r$.

3.2. One Step of the Adiabatic Method. Suppose we are given $V(t) \in C^{r+1}\left(\mathscr{A}_{k}\right)$ and set $H(t):=H_{0}+V(t)$. We wish to diagonalize partially $H(t)$ at every moment $t$ while taking care about differentiability. Partially means starting from some sufficiently high eigen-value and this turns out to be possible owing to the gap condition. Necessarily this subsection is mostly technical but the techniques involved are very standard. This concerns the adiabatic method applied to the lower part of the spectrum and the regular perturbation theory [12] applicable to higher eigen-values. We acquire some basic steps from [8].

We are looking for a family of unitary operators $J(t)$ such that the domain $\mathscr{D}\left(H_{D}(t)\right)=J(t)^{*}(\mathscr{D}(H(t)))$ contains the standard basis and the corresponding matrix $H_{D}(t)=J(t)^{*} H(t) J(t)$ fulfills

$$
\begin{aligned}
H_{D}(t)_{n m} & =0, \quad \text { for } n \leqq N, m>N \text { or } n>N, m \leqq N \\
& =E_{n}(t) \delta_{n m}, \quad \text { for } n>N, m>N
\end{aligned}
$$

Here $N \in \mathbb{N}$ is chosen sufficiently large so that the regular perturbation theory is applicable to $H(t)$ starting from the $N^{t h}$ eigen-value. The eigen-values $\left\{E_{n}(t)\right\}_{n>N}$ form the spectrum of $H(t)$ on the interval ] $\left(E_{N}+E_{N+1}\right) / 2,+\infty$ [. Let us recall briefly the construction.

Set $r_{n}=4^{-1} \min \left\{E_{n}-E_{n-1}, E_{n+1}-E_{n}\right\}$ for $n>1$ and $r_{1}=4^{-1}\left(E_{2}-E_{1}\right) . N$ is chosen so that $r_{n} \geqq 3 \sup \|V(t)\|$, for all $n \geqq N$. Let us denote by $\Gamma_{n}$ the positively oriented circle with radius $r_{n}$ and centred at $E_{n}$ (local notation; $\Gamma_{n}$ shouldn't be confused with the $\Gamma$ operator of the KAM method). Then the projectors $\left(n>N, R(z, t) \equiv(H(t)-z)^{-1}\right)$

$$
P_{n}(t):=-\frac{1}{2 \pi i} \int_{\Gamma_{n}} R(z, t) d z
$$

are rank one and

$$
\varphi_{n}(t)=\left\|P_{n}(t) e_{n}\right\|^{-1} P_{n}(t) e_{n}
$$

are eigen-vectors corresponding to $E_{n}(t)$. They are strongly $C^{r+1}$ and periodic in $t$. Denote by $Q_{0}$ the orthogonal projector onto $\operatorname{span}\left\{e_{1}, \ldots, e_{N}\right\}$ and by $Q(t)$ the spectral projector of $H(t)$ onto the interval ] $\left.-\infty,\left(E_{N}+E_{N+1}\right) / 2\right]$. Then rank $Q(t)=N$. Moreover, using Kato's proof of the adiabatic theorem one can show that there exists a partial isometry $U(t)$ mapping $\operatorname{Ran}\left(Q_{0}\right)$ onto $\operatorname{Ran}(Q(t))$ and vanishing on 
$\operatorname{Ker}\left(Q_{0}\right)=\operatorname{Ran}\left(Q_{0}\right)^{\perp}$. Furthermore, $U(t)$ and $U(t)^{*}$ are periodic in $t$ and strongly $C^{r+1}$. Now we are able to define $J(t)$,

$$
J(t) Q_{0}=U(t), \quad J(t) e_{n}=\varphi_{n}(t), \quad \text { for } n>N .
$$

To be able to cope with differentiability of $J(t)$ we shall need the following lemma which can be extracted from [8], Part I, Sect. 5.

Lemma 3.4. The following estimates hold:

$$
\begin{aligned}
\left|\left\langle\varphi_{n}(t),(d / d t)^{s} \varphi_{n}(t)\right\rangle\right| & \leqq c_{1}(s) n^{-\alpha} \\
\left|\left\langle\varphi_{n}(t),(d / d t)^{s} \varphi_{m}(t)\right\rangle\right| & \leqq c_{2}(s)\left|E_{n}-E_{m}\right|^{-1},
\end{aligned}
$$

for $n, m>N, n \neq m$, and $s=1, \ldots, r+1$.

Now we can state the basic observation of this subsection.

Proposition 3.5. If $V(t)$ is strongly $C^{r+1}$ then the same is true for $J(t)$. Furthermore,

$$
H_{D}(t)-H_{0} \in C^{r+1}\left(\mathscr{A}_{\infty}\right) .
$$

Proof. It is clear from the defining relation (3.5) that $J(t)$ is strongly $C^{r+1}$ on $\operatorname{Ran} Q_{0}$. Thus we have to show the same also for the subspace $\operatorname{Ran}\left(\mathbf{I}-Q_{0}\right)$. Let $\mathscr{L}$ be the linear hull of $\left\{e_{n}\right\}_{n>N}$. Then $\overline{\mathscr{L}}=\operatorname{Ran}\left(\mathbf{I}-Q_{0}\right)$ and $J(t) x$ is $C^{r+1}$ for every $x \in \mathscr{L}$. Denote by $J^{(s)}(t)$ the operator on $\mathscr{L}$ defined by $J^{(s)}(t) x:=(d / d t)^{s} J(t) x$. It suffices to show that the norms $\left\|J^{(s)}(t)\right\|$ are bounded uniformly in $t$ for $s=$ $1, \ldots, r+1$. Instead of $J^{(s)}(t)$ we shall consider two operators $Q_{0} J(t)^{*} J^{(s)}(t)$ and $\left(\mathbf{I}-Q_{0}\right) J(t)^{*} J^{(s)}(t)$. Using repeatedly the identity

$$
Q_{0} J(t)^{*} J^{(j+1)}(t)=-(d / d t) U(t)^{*} \cdot J^{(j)}(t)+(d / d t)\left(Q_{0} J(t)^{*} J^{(j)}(t)\right),
$$

one finds that

$$
Q_{0} J(t)^{*} J^{(s)}(t)=\sum_{j=0}^{s-1} \alpha_{s j} \frac{d^{s-j}}{d t^{s-j}} U(t)^{*} \cdot J^{(j)}(t)
$$

with some constants $\alpha_{s j}$. Thus we deduce that provided $J(t)$ is strongly $C^{s-1}$, then the norm $\left\|Q_{0} J(t)^{*} J^{(s)}(t)\right\|$ is uniformly bounded in $t$. Consequently it is sufficient to show that the norms $\left\|\left(\mathbf{I}-Q_{0}\right) J(t)^{*} J^{(s)}(t)\right\|$ are uniformly bounded in $t$ for $s=1, \ldots, r+1$. But the matrix $\left(Y^{(s)}(t)_{n m}\right)_{n>N, m>N}$ corresponding to $\left(\mathbf{I}-Q_{0}\right) J(t)^{*} J^{(s)}(t)$ restricted to $\operatorname{Ran}\left(\mathbf{I}-Q_{0}\right)$ has entries $Y^{(s)}(t)_{n m}=\left\langle\varphi_{n}(t),(d / d t)^{s}\right.$ $\left.\varphi_{m}(t)\right\rangle$. Thus we can use the estimate (3.7) combined with (3.1) and recall once more the Schur-Holmgren criterion which again leads to the inequalities (3.2), now with $k=1$.

Further we note that from the regular perturbation theory one can deduce that for $n>N$ absolute values of the differences $E_{n}(t)-E_{n}$ are bounded uniformly in $n$ and $t$ and the same is true for the derivatives $(d / d t)^{s} E_{n}(t), s=1, \ldots, r+1$. Since by construction $H_{D}(t)$ fulfills (3.4) the property (3.8) follows immediately.

3.3. Improving Decay of the Perturbation Matrix. The decay property we are interested in is characterized by the class of bounded operators $\mathscr{A}_{k}$ as introduced above. Let us proceed to the formulation of the basic result concerning the adiabatic algorithm. 
Theorem 3.6. Assume that $H_{0}$ acting in a separable Hilbert space $\mathscr{H}$ has a discrete spectrum with simple multiplicity and that the eigen-values fulfill the $\alpha$-gap condition (2.1) with some $\alpha>0$. Assume further that we are given a T-periodic family of potentials $V(t) \in C^{r+1}\left(\mathscr{A}_{k}\right)$, with $k, r \in \mathbb{Z}_{+}$. Then the Floquet Hamiltonian $K=-i \partial_{t}+H_{0}+V(t)$ acting in $\mathscr{K}=L^{2}(] 0, T[, \mathscr{H}, d t)$ (with periodic boundary condition) is unitarily equivalent to $\tilde{K}=-i \partial_{t}+H_{0}+\tilde{V}(t)$, with $\tilde{V}(t) \in C^{r}\left(\mathscr{A}_{k+1}\right)$.

Proof. First we shall show that $J(t) \in C^{r+1}\left(\mathscr{A}_{k+1}\right)$. We know from Lemma 3.5 that $J(t)$ is strongly $C^{r+1}$. The equality $J(t)^{*} H(t) J(t)=H_{D}(t)$ can be rewritten as

$$
\operatorname{ad}\left(H_{0}\right) J(t)=J(t)\left(H_{D}(t)-H_{0}\right)-V(t) J(t) .
$$

Since the RHS of (3.9) is bounded we have $J(t) \in C^{0}\left(\mathscr{A}_{1}\right)$. Now by the fact that $\mathscr{A}_{s}$ is an algebra, by the assumption on $V(t)$ and because of the property (3.8) one can proceed by induction in $s$ to show that $J(t) \in C^{0}\left(\mathscr{A}_{s}\right)$ for $s=1, \ldots, k+1$. Differentiating step by step $(r+1)$-times the relation (3.9) and using the same reasoning in each step one obtains $J(t) \in C^{r+1}\left(\mathscr{A}_{k+1}\right)$.

The family $J(t)$ acting by multiplication in $L^{2}(] 0, T[, \mathscr{H}, d t)$ determines a unitary operator $\mathbf{J}$ in this Hilbert space. One gets $\mathbf{J}^{*} K \mathbf{J}=: \tilde{K}=-i \partial_{t}+H_{0}+\tilde{V}(t)$, where $\left(J^{\prime}(t) \equiv(d / d t) J(t)\right)$

$$
\tilde{V}(t)=H_{D}(t)-H_{0}-i J(t)^{*} J^{\prime}(t)
$$

If $J(t)$ is strongly $C^{r+1}$ then the same is true for $J(t)^{*}$. This fact follows immediately from unitarity (for the zero order) and from the equality $(d / d t) J(t)^{*}=$ $-J(t)^{*} J^{\prime}(t) J(t)^{*}$. Hence $J(t)^{*} \in C^{r+1}\left(\mathscr{A}_{k+1}\right)$ and $J(t)^{*} J^{\prime}(t) \in C^{r}\left(\mathscr{A}_{k+1}\right)$. Thus in view of (3.8) we have $\tilde{V}(t) \in C^{r}\left(\mathscr{A}_{k+1}\right)$.

Applying repeatedly Theorem 3.6 we obtain

Corollary 3.7. With the same assumptions on $H_{0}$, if $V(t)$ is bounded and strongly $C^{r+k}$, with $r, k \in \mathbb{Z}_{+}$, then $K$ is unitarily equivalent to $\tilde{K}$ with $\tilde{V}(t) \in C^{r}\left(\mathscr{A}_{k}\right)$.

Remark. 3.8. Let us now make an observation important in the sequel about dependance of $V$ on an additional parameter $\beta$. Let us assume in Theorem 3.6 that $V(t ; \beta)$ belongs to $C^{r+1}\left(\mathscr{A}_{k}\right)$ in $t$ for all values of $\beta$ lying in some neighbourhood of 0 . We shall require even more, namely that $\beta^{-1} \operatorname{ad}_{H_{0}}^{j} \cdot(d / d t)^{s} V(t, \beta)$ is bounded uniformly for all $t$ and sufficiently small $\beta$ whenever $j=0,1, \ldots, k$, and $s=0,1, \ldots, r+1$. This means that $V(t ; \beta)$ and all its derivatives up to the or$\operatorname{der} r+1$ depend in the norm on $\beta$ as $O(\beta)$. Then the unitary mapping $J(t ; \beta)$ constructed with the help of adiabatic and perturbation methods will possess a similar property: $\beta^{-1}(J(t, \beta)-\mathbf{I})$ and $\beta^{-1}(d / d t)^{j} J(t, \beta)$, for $j=1, \ldots, r+1$, are uniformly bounded in $t$ and $\beta$. The same is true for the operators $\beta^{-1}\left(H_{D}(t ; \beta)-H_{0}\right)$ and $\beta^{-1}(d / d t)^{j} H_{D}(t ; \beta)$, for $j=1, \ldots, r+1$. Now reexamining the proof of Theorem 3.6 one can claim that $\tilde{V}(t ; \beta)$ will depend on $\beta$ in the same manner though with obvious changes in orders related to the new class $C^{r}\left(\mathscr{A}_{k+1}\right)$. Particularly, if we set in Corollary $3.7 V(t ; \beta)=\beta V_{0}(t)$, with $V_{0}(t)$ being strongly $C^{r+k}$, then $\beta^{-1} \mathrm{ad}_{H_{0}}^{j} \cdot(d / d t)^{s} \tilde{V}(t ; \beta)$ will be uniformly bounded in $t$ and $\beta$ for $j=0,1, \ldots, k$, and $s=0,1, \ldots, r$.

Let us conclude this section with some comments concerning absence of the absolutely continuous spectrum and the multiplicity of eigen-values. As observed 
by Howland in [8], if the perturbation $V(t)$ is sufficiently smooth then the absolutely continuous spectrum of the Floquet operator $K$ is empty. Here this result is obtained as a byproduct as demonstrated by

Proposition 3.9. If $X \in \mathscr{A}_{k}$ with $k>\alpha^{-1}$ and $\operatorname{diag}(X)=0$ then $X$ is trace class.

Proof. The proof follows from the estimate $\|X\|_{\text {trace }} \leqq \sum_{n, m}\left|X_{n m}\right|$. The RHS can be shown to be finite with the help of the integral criterion.

We recall too that the condition on zero diagonal means no principal restriction on $\tilde{V}(t)$ since $\operatorname{diag} \tilde{V}(t)$ can be written as a sum of a bounded constant part plus a time dependent part but with the time average equal to zero. The constant part can be combined with $H_{0}$ and the time dependent part can be removed by a gauge transformation [8].

Remark. Note that the assumption about simplicity of the spectrum of $H_{0}$ was needed in fact only in some neighbourhood of infinity. But we even suggest that this version of treating the adiabatic process can be extended to the more general case considered by Nenciu [14] and Joye [10]. Namely, rather than imposing the $\alpha$-gap condition on eigen-values of $H_{0}$ one assumes that the spectrum of $H_{0}$ can be written as a union of groups of eigen-values, $\sigma\left(H_{0}\right)=\bigcup \sigma_{n}$, with multiplicities uniformly bounded and the $\alpha$-gap condition is then imposed on these groups of eigen-values, $\operatorname{dist}\left(\sigma_{n}, \sigma_{n+1}\right) \geqq c n^{\alpha}$. Apparently the main complication caused by this generalization is that infinite matrices should be split into finite-dimensional blocks which may lead to a more complicated notation.

\section{Pure Point Spectrum}

4.1. Main Theorem. Now we can formulate the main result of this paper. The Floquet Hamiltonian will be assumed to depend also on a real coupling constant $\beta, K_{\omega, \beta}=-i \omega \partial_{t}+H_{0}+\beta V(t)$. The conditions on $H_{0}$ are the same as in the previous two sections, $V(t)$ is $2 \pi$-periodic and strongly $C^{r+k}$, with $r, k \in \mathbb{Z}_{+}$ to be determined. According to Corollary 3.7 and Remark 3.8, after $k$ steps of the adiabatic method we get another but unitarily equivalent Floquet Hamiltonian $\tilde{K}_{\omega, \beta}=-i \omega \partial_{t}+H_{0}+\tilde{V}(t ; \beta)$, with $\tilde{V}(t ; \beta) \in C^{r}\left(\mathscr{A}_{k}\right)$ and, in the sense specified in Remark $3.8, \tilde{V}(t ; \beta)$ is of order $O(\beta)$. We shall suppress the tilde.

The symbols $V_{m n}(t ; \beta)$ stand for matrix elements in the eigen-basis of $H_{0}$. Then for $i, j \in \mathbb{Z} \times \mathbb{N}$,

$$
V(i, j ; \beta)=\frac{1}{2 \pi} \int_{0}^{2 \pi} V_{i_{2} j_{2}}(t ; \beta) \mathrm{e}^{-\mathrm{i}\left(i_{1}-j_{1}\right) t} d t .
$$

Integrating $r$-times by parts and using $V \in C^{r}\left(\mathscr{A}_{k}\right)$ we get

$$
\left|E_{i_{2}}-E_{j_{2}}\right|^{k}|V(i, j ; \beta)| \leqq c^{\prime}|\beta|\left(1+\left|i_{1}-j_{1}\right|\right)^{-r} .
$$

Since, by virtue of (3.1),

$$
\left|E_{n}-E_{m}\right| \geqq c^{\prime \prime}\left|n^{1+\alpha}-m^{1+\alpha}\right| \geqq c^{\prime \prime}|n-m|^{1+\alpha},
$$

we arrive finally at the estimate

$$
|V(i, j ; \beta)| \leqq c|\beta|\left(1+\left|i_{1}-j_{1}\right|\right)^{-r}\left(1+\left|i_{2}-j_{2}\right|\right)^{-(1+\alpha) k} .
$$


Now to apply Theorem 2.6 we need $C_{V}$ to be finite (cf. (2.18)). A sufficient condition is that both $r$ and $(1+\alpha) k$ are strictly greater than $\tau_{\star}(\alpha)$. In the final formulation of the result we shall rescale back the time.

Theorem 4.1. Assume that $H_{0}$ acting in a separable Hilbert space $\mathscr{H}$ has a simple discrete spectrum and its eigen-values $\left\{E_{n}\right\}$ fulfill the $\alpha$-gap condition (2.1) with some $\alpha>0$. Assume further that there is given a $2 \pi$-periodic strongly continuous family $V(t)$ of self-adjoint bounded operators in $\mathscr{H}$. The Floquet Hamiltonian $K_{\omega, \beta}:=-i \partial_{t}+H_{0}+\beta V(\omega t)$ is supposed to act in $\mathscr{K}=L^{2}(] 0, T[, \mathscr{H}, d t)$, $T=2 \pi / \omega$, with periodic boundary condition in $t$ and with the frequency $\omega$ lying in an interval $\Omega=[a, b], 0<a<b<\infty$.

If $V(t)$ is $N(\alpha)$-times strongly differentiable,

$$
N(\alpha):=\left[\tau_{\star}(\alpha)\right]+\left[\tau_{\star}(\alpha) /(1+\alpha)\right]+2,
$$

$\left(\tau_{\star}(\alpha)\right.$ is given by (2.27)) then there exist constants $\gamma^{\star}>0, \beta^{\star}>0$, such that the inequalities

$$
0<\gamma<\gamma^{\star}, \quad 0 \leqq \beta \leqq \beta^{\star} \gamma^{2},
$$

imply that one can find $\Omega^{\prime} \equiv \Omega^{\prime}(\gamma, \beta) \subset \Omega$ with properties

$$
\left|\Omega \backslash \Omega^{\prime}\right|<\gamma \text { and } K_{\omega, \beta} \text { is pure point }
$$

for every $\omega \in \Omega^{\prime}$.

4.2. Example: Fermi Accelerator. To give at least one illustration to Theorem 4.1 let us mention the well known Fermi accelerator. In this case the Hilbert space itself depends on $t, \mathscr{H}_{t}=L^{2}(] 0, y(t)[, d x)$, with $y(t)$ being a $T$-periodic strictly positive function. The time-dependent Hamiltonian is $H(t)=-d^{2} / d x^{2}$ wih Dirichlet boundary conditions. The Floquet Hamiltonian acts in $\mathscr{K}=L^{2}(M, d t d x), M=$ $\{(t, x) ; 0<t<T, 0<x<y(t)\}$, and is defined as $K=-i \partial_{t}-\partial_{x}^{2}$, with periodic boundary conditions in $t$ and Dirichlet boundary conditions in $x$.

$K$ is known to be self-adjoint [11,9]. To treat the spectral problem of $K$ we shall borrow a transformation procedure from the paper [15]. First one applies the unitary transformation

$$
\begin{aligned}
& \mathbf{W}: L^{2}(M, d t d x) \rightarrow L^{2}(] 0, T[, d t) \otimes L^{2}(] 0,1[, d x), \\
& (\mathbf{W} \psi)(t, x)=y(t)^{1 / 2} \exp \left(-\frac{i}{4} y(t) y^{\prime}(t) x^{2}\right) \psi(t, y(t) x),
\end{aligned}
$$

to get

$$
K_{1}:=\mathbf{W} K \mathbf{W}^{-1}, \quad K_{1}=-i \partial_{t}+y(t)^{-2}\left(H_{0}+V(t)\right),
$$

where $-H_{0}$ is the Dirichlet Laplacian in $L^{2}(] 0,1[, d x)$ and $V(t)$ is a family of bounded operators in the same Hilbert space,

$$
V(t)=\frac{1}{4} y(t)^{3} y^{\prime \prime}(t) x^{2}
$$

Furthermore, the function

$$
f(t):=\int_{0}^{t} d s y(s)^{-2}
$$


fulfills $f(t+T)=f(t)+\tilde{T}$, with $\tilde{T}:=f(T)$, and conversely, $f^{-1}(t+\tilde{T})=f^{-1}(t)$ $+T$. Set

$$
\tilde{K}:=-i \partial_{t}+H_{0}+\tilde{V}(t), \quad \tilde{V}(t):=V\left(f^{-1}(t)\right),
$$

acting in $L^{2}(] 0, \tilde{T}[, d t) \otimes L^{2}(] 0,1[, d x)$. Denote by $U_{1}(t)$ and $\tilde{U}(t)$ the 1-parameter families of unitary operators in $L^{2}(] 0,1[, d x)$ determined by $U_{1}(0)=\tilde{U}(0)=\mathbf{I}$ and

$$
i \partial_{t} U_{1}(t)=y(t)^{-2}\left(H_{0}+V(t)\right) U_{1}(t), \quad i \partial_{t} \tilde{U}(t)=\left(H_{0}+\tilde{V}(t)\right) \tilde{U}(t) .
$$

It holds $\tilde{U}(t)=U_{1}\left(f^{-1}(t)\right)$, particularly $\tilde{U}(\tilde{T})=U_{1}(T)$. The families $U_{1}(t)$ and $\tilde{U}(t)$ induce unitary operators $\mathbf{U}_{1}$ and $\tilde{\mathbf{U}}$ in the corresponding (obvious) tensor products of Hilbert spaces and, as shown by Yajima [17] and Howland [7],

$$
\mathrm{e}^{-i K_{1} T}=\mathbf{U}_{1}\left(\mathbf{I} \otimes U_{1}(T)\right) \mathbf{U}_{1}^{-1}, \quad \mathrm{e}^{-i \tilde{K} \tilde{T}}=\tilde{\mathbf{U}}(\mathbf{I} \otimes \tilde{U}(\tilde{T})) \tilde{\mathbf{U}}^{-1}
$$

Consequently, $K$ is pure point if and only if the same is true for $\tilde{K}$. So one can focus on the Hamiltonian $H_{0}+\tilde{V}(t)$ instead of the original one. But now, $\tilde{V}(t)$ is a $\tilde{T}$-periodic family of bounded operators which is, moreover, strongly $C^{r}$ if $y$ is chosen $C^{r+2}$.

It was conjectured in [9] that in the generic case $K$ is pure point. We can confirm this conjecture provided $y$ is sufficiently smooth, the amplitude of oscillation is small and the frequency is non-resonant. To be more precise assume that

$$
y(t)=y_{0}+\beta z(\omega t),
$$

$z$ is $2 \pi$-periodic and $\omega \in \Omega=[a, b], 0<a<b<\infty$. From the above transformation it follows (cf. (4.3)) that $\tilde{V}(t ; \beta)$ is of order $O(\beta)$ and strongly $C^{r}$ provided $z \in C^{r+2}$. In this case $H_{0}$ fulfills the $\alpha$-gap condition with $\alpha=1$. Recall that $N(1)=17$ (cf. (4.2)).

Now we can apply Theorem 4.1.

Proposition 4.2. If $z \in C^{19}$ then there exist constants $\gamma^{\star}>0$ and $\beta^{\star}>0$ such that the inequalities

$$
0<\gamma<\gamma^{\star}, \quad 0 \leqq \beta \leqq \beta^{\star} \gamma^{2}
$$

imply that one can find $\Omega^{\prime} \equiv \Omega^{\prime}(\gamma, \beta) \subset \Omega$ with properties: $\left|\Omega \backslash \Omega^{\prime}\right|<\gamma$ and for each $\omega \in \Omega^{\prime}$, the Floquet Hamiltonian $K$ related to the Fermi accelerator is pure point.

Remark. We claim that the condition $z \in C^{19}$ in this proposition can be weakened to $z \in C^{18}$. The point is that the regularized potential $\tilde{V}(t)$ itself exhibits some decay. Assuming that $z \in C^{18}$ we have $\tilde{V}(t)=v(t) x^{2}$, with $v \in C^{16}$. One can calculate explicitly the matrix elements of the potential $x^{2}$ in the eigen-basis of $H_{0}$. The eigen-vectors are $\varphi_{n}(x)=\sqrt{2} \sin (n \pi x)$, the eigen-values $E_{n}=n^{2} r^{2}, n \in \mathbb{N}$, and the resulting matrix elements equal $(-1)^{m+n} 8 m n / \pi^{2}\left(m^{2}-n^{2}\right)^{2}$, for $m \neq n$. Consequently,

$$
\sup _{m, n}|n-m|^{\beta}\left|(d / d t)^{s} \tilde{V}_{n, m}(t)\right|<\infty,
$$

with $\beta=2$ and $0 \leqq s \leqq 16$. After five steps of the adiabatic algorithm we obtain a transformed potential, $\tilde{V}(t)$, which is strongly $C^{11}$ and obeys a similar condition but now with $\beta=12$ and $0 \leqq s \leqq 11$. This property is already sufficient for the application of the KAM-type iteration as claimed in Theorem 2.6. 
We note that it is possible to pass from this observation to a general assertion but we restrict ourselves to just a sketchy remark. One can introduce another type of classes of operators $\mathscr{C}_{\beta} \subset \mathscr{B}\left(l^{2}\right)$, in addition to those having been introduced in Definition 3.1. An operator $X \in \mathscr{B}\left(l^{2}\right)$ belongs to $\mathscr{C}_{\beta}$ if and only if its matrix elements $\left(X_{n m}\right)$ in the standard basis obey the condition

$$
\sup _{n, m}|n-m|^{\beta}\left|X_{n, m}\right|<\infty \text {. }
$$

It is not difficult to verify that $\mathscr{C}_{\beta}$ is a $*$-subalgebra of $\mathscr{B}(l)^{2}$ provided $\beta>1$ (or $\beta=0$ ). Clearly, $\mathscr{C}_{0}=\mathscr{B}\left(l^{2}\right)$ and $\mathscr{A}_{k} \subset \mathscr{C}_{k(1+\alpha)}$ (and is not equal), for $k=$ $1,2 \ldots$ Let us also define the symbol $C^{r}\left(\mathscr{C}_{\beta}\right) \equiv \mathscr{C}_{\beta}^{r}$ in a way quite analogous to Definition 3.3. Adapting the proof of Theorem 3.6 one can show that the final assertion of this theorem can be reformulated as

Assume further that we are given a T-periodic family of potentials $V(t) \in \mathscr{C}_{\beta}^{r+1}$, with $r \in \mathbb{Z}_{+}, \beta=0$ or $\beta>1$. Then the Floquet Hamiltonian $K=-i \partial_{t}+H_{0}+$ $V(t)$ acting in $\mathscr{K}=L^{2}(] 0, T[, \mathscr{H}, d t)$ is unitarily equivalent to $\tilde{K}=-i \partial_{t}+H_{0}+$ $\tilde{V}(t)$, with $\tilde{V}(t) \in \mathscr{C}_{\beta+\alpha+1}^{r}$.

\section{Appendix 1. Proof of Theorem 2.1}

Since obviously $I_{k, m, n}=I_{-k, n, m}$ it is sufficient to consider only non-negative $k$. Where convenient we shall reparameterize $(k, m, n)$ as

$$
p:=m-n \quad \text { and } \quad d:=k+m-n=k+p .
$$

Proof will be done in several steps.

Step 1. Excluding the cases $k=0$ and $n=m$. One has obviously for all $n \neq m$,

$$
\begin{aligned}
& \Delta E>\|g\|_{0} \text { and } \gamma \leqq \Delta E-\|g\|_{0} \Rightarrow \\
& \quad \forall \omega \in \Omega,\left|E_{n}-E_{m}+g(n, m ; \omega)\right| \geqq \gamma \geqq \gamma|n-m|^{-\sigma} \Rightarrow I_{0, n, m}=\emptyset .
\end{aligned}
$$

Similarly for all $k \neq 0$,

$$
\gamma \leqq \inf \Omega \Rightarrow \forall \omega \in \Omega, \quad|k \omega| \geqq \inf \Omega \geqq \gamma|k|^{-\sigma} \Rightarrow I_{k, n, n}=\emptyset .
$$

Moreover, notice that $I_{k, m, n}=\emptyset$ as soon as $n \geqq m$. From now on we shall only consider $k \geqq 1$ and $1 \leqq n<m$, whence $1 \leqq p \leqq d-1$.

Step 2. Measuring the $I_{k, m, n}$. Let $\omega$ and $\omega^{\prime}$ be in $I_{k, m, n}$ and denote $g(n, m ; \omega)$ and $g\left(n, m ; \omega^{\prime}\right)$ shortly by $g$ and $g^{\prime}$. Then one has

$$
\begin{aligned}
2 \gamma|k+m-n|^{-\sigma} & >\left|k\left(\omega-\omega^{\prime}\right)+g-g^{\prime}\right|=\left|\omega-\omega^{\prime}\right|\left|k+\frac{g-g^{\prime}}{\omega-\omega^{\prime}}\right| \\
& \geqq\left|\omega-\omega^{\prime}\right|\left(k-\|g\|_{1}\right) .
\end{aligned}
$$

Since $\|g\|_{1}<1$, we find

$$
\left|I_{k, m, n}\right| \leqq \frac{2 \gamma d^{-\sigma}}{d-p-\|g\|_{1}}
$$


Step 3. Counting the $I_{k, m, n}$. If $I_{k, m, n}$ is not empty, this means that there exists $\omega$ such that, on the one hand, $(b=\sup \Omega)$

$$
\begin{aligned}
E_{m}-E_{n} & =-\left(k \omega+E_{n}-E_{m}+g\right)+k \omega+g<\gamma d^{-\sigma}+k \omega+\|g\|_{0} \\
& \leqq \gamma+(d-1) \omega+\|g\|_{0} \leqq d \omega \leqq d b .
\end{aligned}
$$

On the other hand, owing to the gap condition (2.1) with $\alpha>0$ (it is crucial now), there exists $C_{E}>0$ such that

$$
C_{E}\left(n^{\alpha}+m^{\alpha}\right) \leqq \frac{E_{m}-E_{n}}{m-n} .
$$

Combining (A1.2) and (A1.3) we get for fixed $p$ and $d$,

$$
1+(n+p)^{\alpha} \leqq n^{\alpha}+(n+p)^{\alpha} \leqq \frac{b}{C_{E}} \frac{d}{p} .
$$

Set

$$
r:=b / C_{E}
$$

(only local notation, for purpose of this proof). Then we deduce that $p \leqq(r d)^{1 /(1+\alpha)}$ and for given $d$ and $p$ there are at most $((r d / p)-1)^{1 / \alpha}-p$ values of $n$. Finally one can see easily that $d>\left(2^{\alpha}+1\right) / r$.

Step 4. Upper bound on the measure of $\Omega_{\mathrm{bad}}$. According to the above discussion (cf. (A1.1) and the estimate of number of $n$ 's) the measure of $\Omega_{\mathrm{bad}}$ is bounded by

$$
\sum_{d=d_{\min }}^{\infty} \sum_{p=1}^{p_{\max }(d)}\left(\left(\frac{r d}{p}\right)^{1 / \alpha}-p\right) \frac{2 \gamma d^{-\sigma}}{d-p-\|g\|_{1}},
$$

where $p_{\max }(d):=\min \left\{\left[(r d)^{1 /(1+\alpha)}\right], d-1\right\}$ and $d_{\min }:=\left[\left(2^{\alpha}+1\right) / r\right]$.

Further we shall use the estimate

$$
\left(d-p-\|g\|_{1}\right)^{-1} \leqq \frac{2}{1-\|g\|_{1}} \max \left\{1,(2 r)^{\frac{1}{\alpha}}\right\} \frac{1}{d} .
$$

To see (A1.5) it is enough to notice that $d-p-\|g\|_{1} \geqq(d-p)\left(1-\|g\|_{1}\right)$ and afterwards to consider separately the cases $d \leqq 2(2 r)^{1 / \alpha}$ and $d \geqq 2(2 r)^{1 / \alpha}$.

Next, replacing $p_{\max }(d)$ by $\left[(r d)^{1 /(1+\alpha)}\right]$ and using $\sum_{1}^{[y]} p^{-1 / \alpha} \leqq 1+\int_{1}^{y} p^{-1 / \alpha} d p$, we can estimate

$$
\sum_{p=1}^{p_{\max }(d)}\left(\frac{r d}{p}\right)^{\frac{1}{\alpha}}-p \leqq \varphi_{\alpha}(r d)
$$

where

$$
\varphi_{\alpha}(x):=\frac{x^{1 / \alpha}}{\alpha-1}\left(\frac{\alpha+1}{2} x^{(\alpha-1) / \alpha(\alpha+1)}-1\right) .
$$

In the case $\alpha=1$ a simple limit shows that $\varphi_{1}(x)=(x / 2) \ln (x)$.

Finally it remains to perform the summation over $d$. Taking into account the asymptotic of $\varphi_{\alpha}(x), x \rightarrow+\infty$, one finds that $\sigma$ must be chosen larger than $\sigma_{\star}(\alpha)$ (see (2.12) for its definition) in order to insure the convergence of the sum. The summation of $\varphi_{\alpha}(r d) d^{-1-\sigma}$ will give a constant which depends on $r, \alpha$ and $\sigma$ and 
since $r$ depends on $E$ and $\Omega$ (in fact, only on $\sup \Omega$ ) we conclude that

$$
\left|\Omega_{\mathrm{bad}}\right| \leqq \frac{C_{1}(E, \Omega, \sigma)}{1-\|g\|_{1}} \gamma
$$

\section{Appendix 2. Proof of Theorem 2.3}

We define $g(n, m ; \omega):=G(n ; \omega)-G(m ; \omega)$. Clearly, both $\|g\|_{i}, i=0,1$, are estimated by $2\|G\|_{\Omega}$ and the condition (2.9) is fulfilled. Moreover, since $2\|G\|_{\Omega}<$ $\min \{1, \Delta E$, inf $\Omega\}$, the conditions (2.10) and (2.11) are satisfied as well.

Let $W=\Gamma V$. The estimate of $\|W\|_{\Omega^{\prime} r^{\prime}}$ is rather technical; to avoid cumbersome formulas we introduce temporarily the shorter notations

$$
d:=|i-j|, \quad \delta:=D_{0}(i ; \omega)-D_{0}(j ; \omega)+g\left(i_{2}, j_{2} ; \omega\right), \quad V:=V(i, j ; \omega),
$$

with the same convention for $W$ and $g$. Furthermore, $\delta^{\prime}, V^{\prime}, \ldots$, designate $\delta, V, \ldots$, where $\omega$ has been replaced by $\omega^{\prime}$. Since all conditions to apply Theorem 2.1 were verified there exists a subset $\Omega^{\prime}$ of $\Omega$ so that for all $(i, j, \omega) \in(\mathbb{Z} \times \mathbb{N})^{2} \times \Omega^{\prime}$ it holds $|\delta| \geqq \gamma d^{-\sigma}$ (and similarly for $\delta^{\prime}$ ).

Then we have $W=V / \delta, W^{\prime}=V^{\prime} / \delta^{\prime}$,

$$
|W|+\left|\frac{W-W^{\prime}}{\omega-\omega^{\prime}}\right| \leqq\left|\frac{V}{\delta}\right|\left(1+\left|\frac{1}{\delta^{\prime}} \frac{\delta-\delta^{\prime}}{\omega-\omega^{\prime}}\right|\right)+\left|\frac{1}{\delta^{\prime}} \frac{V-V^{\prime}}{\omega-\omega^{\prime}}\right|
$$

and

$$
\left|\frac{\delta-\delta^{\prime}}{\omega-\omega^{\prime}}\right|=\left|i_{1}-j_{1}+\frac{g-g^{\prime}}{\omega-\omega^{\prime}}\right| \leqq d+2\|G\|_{\Omega} .
$$

Because $d \geqq 1$ and $\gamma \leqq \gamma_{0}:=\min \{\Delta E$, inf $\Omega\}-2\|G\|_{\Omega}$ we get

$$
1+\left|\frac{1}{\delta^{\prime}} \frac{\delta-\delta^{\prime}}{\omega-\omega^{\prime}}\right| \leqq 1+d^{\sigma} \gamma^{-1}\left(d+2\|G\|_{\Omega}\right) \leqq \gamma^{-1} d^{\sigma+1}\left(\gamma_{0}+1+2\|G\|_{\Omega}\right) .
$$

Consequently,

$$
|W|+\left|\frac{W-W^{\prime}}{\omega-\omega^{\prime}}\right| \leqq \gamma^{-2} d^{2 \sigma+1}\left(1+\gamma_{0}+2\|G\|_{\Omega}\right)\left(|V|+\left|\frac{V-V^{\prime}}{\omega-\omega^{\prime}}\right|\right)
$$

and

$\sup _{|i-j|=d ; \omega, \omega^{\prime} \in \Omega^{\prime}}\left(|W|+\left|\frac{W-W^{\prime}}{\omega-\omega^{\prime}}\right|\right) \leqq c_{2} \gamma^{-2} d^{2 \sigma+1} \sup _{|i-j|=d ; \omega, \omega^{\prime} \in \Omega^{\prime}}\left(|V|+\left|\frac{V-V^{\prime}}{\omega-\omega^{\prime}}\right|\right)$,

with $c_{2}:=1+\min \{\Delta E$, inf $\Omega\}$.

It remains to sum over $d$. Since for positive $\rho$ :

$$
\sup _{d \geqq 0} \mathrm{e}^{-\rho d} d^{2 \sigma+1}=\left(\frac{2 \sigma+1}{\mathrm{e} \rho}\right)^{2 \sigma+1},
$$

the theorem is proved. The bound on $\left|\Omega \backslash \Omega^{\prime}\right|$ is just that one given by Theorem 2.1. 
Acknowledgements. P.S. wishes to express his gratitude to his hosts at the Centre de Physique Théorique of Marseille Luminy and Université de Toulon et du Var where this work was initiated and finished. Partial support from Grant No. 201/94/0708 of Czech GA is also gratefully acknowledged. P.D. thanks also the Faculty of Nuclear Sciences of CTU for its hospitality during the process of this work.

\section{References}

1. Bellissard, J.: Stability and instability in quantum mechanics. In: Trends and Developments in the Eighties, Albeverio, S., Blanchard, Ph. (eds) Singapore: World Scientific, 1985, pp. 1-106

2. Bleher, P.M., Jauslin, H.R., Lebowitz, J.L.: Floquet operators for two-level systems in quasiperiodic time dependent fields. J. Stat. Phys. 68, 271 (1992)

3. Combes, J.-M.: Connection between quantum dynamics and spectral properties of timeevolution operators. In: Differential Equations and Applications to Mathematical Physics, Ames, W.F. et al. (eds.) Mathematics in Sciences and Engineering 192, New York, London: Academic Press, 1993, pp. 59-68

4. Combes, J.-M., Hislop, P.D., Mourre, E.: Spectral averaging, perturbation of singular spectrum, and localization. Preprint Vienna ESI 124 (1994)

5. Combescure, M.: The quantum stability problem for time-periodic perturbations of the harmonic oscillator. Ann. Inst. Henri Poincaré 47, 62-82 (1987); Erratum: Ann. Inst. Henri Poincaré 47, 451-454 (1987)

6. Enss, V., Veselić, K.: Bound states and propagating states for time-dependent Hamiltonians. Ann. Inst. Henri Poincaré 39, 159-191 (1983)

7. Howland, J.S.: Scattering theory for Hamiltonians periodic in time. Indiana J. Math. 28, 471-494 (1979)

8. Howland, J.S.: Floquet operators with singular spectrum I. Ann. Inst. Henri Poincaré 49, 309-323 (1989); Floquet operators with singular spectrum II. Ann. Inst. Henri Poincaré 49, 325-334 (1989)

9. Howland, J.S.: Stability of Fermi accelerator. Unpublished notes (1992)

10. Joye, A.: Absence of absolutely continuous spectrum of Floquet operators. J. Stat. Phys. 75, 929-952 (1994)

11. Karner, B.: On the quantum Fermi accelerator and its relevance to "quantum chaos", Lett. Math. Phys. 17, 329-339 (1989)

12. Kato, T.: Perturbation theory of linear operators. Berlin, Heidelberg, New York: Springer, 1966

13. Kotani, S.: Ljapunov indices determine absolutely continuous spectra of stationary random onedimensional Schrödinger operators. In: Stochastic Analysis. Ito, K. (ed.) Amsterdam: North Holland, 1984, pp. 225-248

14. Nenciu, G.: Floquet operators without absolutely continuous spectrum. Ann. Inst. Henri Poincaré 59, 91-97 (1993)

15. Seba, P.: Quantum chaos in Fermi-accelerator model. Phys. Rev. A 41, 2306-2310 (1990)

16. Simon, B., Wolf, T.: Singular continuous spectrum under rank one perturbation and localization for random Hamiltonians. Comm. Pure Appl. Math. 39, 75-90 (1986)

17. Yajima, K.: Scattering Theory for Schrödinger Equations with Potential Periodic in Time. J. Math. Soc. Japan 29, 729-743 (1977) 
